SLAC - PUB - 3812

October 1985

(T/AS)

\title{
QUANTUM INSTABILITY OF DE SITTER SPACE*
}

\author{
I. ANTONIADIS ${ }^{*}$ \\ Stanford Linear Accelerator Center \\ Stanford University, Stanford, California, 94305 \\ J. ILIOPOULOS ${ }^{\dagger}$ \\ Laboratoire de Physique Theorique \\ Ecole Normale Superieure, Paris, France \\ and \\ T. N. TOMARAS ${ }^{\ddagger}$ \\ Physics Department \\ University of Crete, Iraklion, Crete, Greece
}

\begin{abstract}
The graviton propagator in a de Sitter background is found to be divergent. We show that as a consequence of this divergence, de Sitter space is not a solution of the equations of motion of the complete theory. If we start from de Sitter space as a classical ground state, quantum corrections change it into flat Minkowski.
\end{abstract}

Submitted to Physical Review Letters

* Work supported by the Department of Energy, contract DE - A C03 - 76SF00515.

* On leave of absence from Centre de Physique Theorique, Ecole Polytechnique, Palaiseau, France.

† Laboratoire propre du CNRS associé à l'Ecole Normale Superieure et à l'Université de Paris Sud.

$\ddagger$ Supported in part by the Physics Research Institute of Crete. 
An outstanding problem in the theory of gravitation is the observed vanishingly small value of the cosmological constant. ${ }^{1}$ In perturbation theory this parameter can assume any value and one has to fine-tune it to zero in order to have a vacuum state corresponding to flat Minkowski space. Several attempts have been made to argue that the zero-value may be preferred dynamically ${ }^{2,3}$ and in this letter we want to discuss a new approach along this line. We shall present only our method and results and we shall leave all technical details for a lengthier publication. ${ }^{4}$ Although all our formulae and conclusions can be trivially extended to $d$-dimensions, in this letter we shall restrict ourselves to $d=4$.

Our starting point is the observation that the propagator of a massless scalar field in four-dimensional de Sitter space is singular. ${ }^{5}$ To be more precise, let us consider a scalar field described by:

$$
\mathcal{L}=\frac{1}{2} \sqrt{-g}\left\{g^{\mu \nu}\left(\partial_{\mu} \varphi\right)\left(\partial_{\nu} \varphi\right)-m^{2} \varphi^{2}\right\}
$$

We choose the background metric of the form:

$$
g_{\mu \nu}=\bar{g}_{\mu \nu} \equiv\left(t^{2} a^{2}\right)^{-1} \eta_{\mu \nu}
$$

with $\eta_{\mu \nu}$ the flat Minkowski metric. The Lagrangian (1), after some algebra and a field rescaling, becomes:

$$
\mathcal{L}=\frac{1}{2} \varphi D^{-1} \varphi
$$

with

$$
D^{-1}=-\partial_{0}^{2}-\vec{k}^{2}+\frac{1}{t^{2}}\left(2-\frac{m^{2}}{a^{2}}\right) .
$$

The inverse of the operator $D^{-1}$ depends on the initial conditions one chooses to impose, but for a choice which preserves the symmetries of de Sitter space, ${ }^{5} D$ 
is given by:

$$
D\left(\vec{x}, t ; \vec{x}^{\prime}, t^{\prime}\right)=\frac{1}{t t^{\prime}} \frac{\Gamma\left(\frac{3}{2}-\nu\right) \Gamma\left(\frac{3}{2}+\nu\right)}{(4 \pi)^{2}} F\left[\frac{3}{2}-\nu, \frac{3}{2}+\nu, 2 ; 1+\frac{a^{2} \sigma^{2}}{4}\right]
$$

where $\sigma^{2}$ is the invariant distance $\sigma^{2}=\left(a^{2} t t^{\prime}\right)^{-1}\left[\left(t-t^{\prime}\right)^{2}-\left(\vec{x}-\vec{x}^{\prime}\right)^{2}\right]$, $\nu=\left(\frac{9}{4}-\frac{m^{2}}{a^{2}}\right)^{1 / 2}$ and $F$ is a hypergeometric function. We see that for $m^{2} \rightarrow 0$ the propagator develops a pole of the form $1 / \mathrm{m}^{2}$.

Our argument will be based on the fact that the same pathology occurs also for the graviton propagator in de Sitter background. Let us first prove this statement. We start with the Lagrangian:

$$
\mathcal{L}=\sqrt{-g}\left(-\frac{2}{\kappa^{2}} R+\Lambda\right)
$$

We expand around the background given by Eq. (2) and we write:

$$
g_{\mu \nu}=\bar{g}_{\mu \nu}+h_{\mu \nu}
$$

The scalar curvature of the background is:

$$
\bar{R}=-12 a^{2}=\Lambda \kappa^{2}
$$

We must now choose a gauge, isolate in (5) the quadratic part and solve the corresponding equation of motion in order to find the graviton propagator. This is quite lengthy and tedious, so we try to find a gauge in which the differential equation reduces to that of the scalar field whose properties have been studied in the literature. We found it convenient to have as much gauge freedom as possible 
and we promote the Lagrangian (5) into a locally conformally invariant one by introducing a scalar compensating field $\Phi(x)$.

$$
\mathcal{L}=\sqrt{-g}\left\{-\frac{1}{2}\left(\partial_{\mu} \varphi\right)\left(\partial_{\nu} \varphi\right) g^{\mu \nu}-\frac{1}{12} R(V+\varphi)^{2}+\lambda(V+\varphi)^{4}\right\}
$$

where conformal invariance is spontaneously broken by the vacuum expectation value of $\Phi(x)$ which we wrote as $\varphi(x)+V$ with $V=2 \sqrt{6} / \kappa$. The coupling constant $\lambda$ is equal to $\Lambda \kappa^{4}(24)^{-2}$. We wrote the Lagrangian (8) in four dimensions but it can be extended to arbitrary $d$, still preserving local conformal invariance. ${ }^{6,7}$ Now we can impose five gauge conditions and we take them to be:

$$
h_{0 \mu}=0 \quad ; \quad h_{i}^{i}=0
$$

It is straightforward to compute the graviton propagator in this gauge. We must expand (8) according to (6) and keep only terms quadratic in the fields. After some algebra, diagonalizing the $h-\varphi$ terms and rescaling the fields we obtain

$$
\mathcal{L} \sim \frac{1}{2} h^{i j} D_{i j, k \ell}^{-1} h^{k \ell}
$$

with

$$
D_{i j, k \ell}^{-1}=\lim _{\xi \rightarrow 0}\left\{\left[-\partial_{0}^{2}-\vec{k}^{2}+\frac{2}{t^{2}}\right] \eta_{i k} \eta_{j \ell}-2 \vec{k}^{2} \eta_{i k} \omega_{j \ell}+\xi^{-1} \eta_{i j} \eta_{k \ell}\right\}
$$

where $\omega_{i j}=k_{i} k_{j} / \vec{k}^{2}$. The last term proportional to $\xi^{-1}$ is due to the fifth gauge condition $h_{i}^{i}=0$. We can invert the operator (10) by introducing the complete set of 3-dimensional projectors for symmetric rank-4 tensors $P^{(2)}, P^{(1)}, P^{(0-s w)}$, $P^{(0-w s)}, P^{(0-s)}, P^{(0-w)}$ whose precise definition is given in Refs. 8 and 7 . We 
only notice that $P^{(2)}$ is the projector for the transverse and traceless, spin-two part. In terms of these operators we can show that:

$$
\begin{aligned}
& {\left[A P^{(2)}+B P^{(1)}+C P^{(0-s)}+D\left(P^{(0-s w)}+P^{(0-w s)}\right)+E P^{(0-w)}\right]^{-1} } \\
= & \frac{1}{A} P^{(2)}+\frac{1}{B} P^{(1)}-\frac{E}{D^{2}-C E} P^{(0-s)}+\frac{D}{D^{2}-C E}\left(P^{(0-s w)}+P^{(0-w s)}\right) \\
& -\frac{C}{D^{2}-C E} P^{(0-w)} .
\end{aligned}
$$

In our case the operator (10) is of the form of the l.h.s. of (11) with the identification

$$
\begin{aligned}
& A=-\partial_{0}^{2}-\vec{k}^{2}+\frac{2}{t^{2}} ; \quad B=A-\vec{k}^{2}, \quad C=A+2 \xi^{-1} \\
& D=\sqrt{2} \xi^{-1} ; \quad E=A-2 \vec{k}^{2}+\xi^{-1} .
\end{aligned}
$$

We see that $A$, whose inverse is precisely the coefficient of the transverse part of the propagator, equals the operator given by (3) with $m^{2}=0 .{ }^{9}$ It follows that, at least this part, contains the singularity of the massless scalar field. This result is valid in all dimensions. How are we sure that this result is not an artifact of the special gauge we have chosen? First we notice that, since the singularity appears -in the transverse part of the graviton propagator, it cannot be removed by a change of gauge. However, the real proof of the relevance of this effect consists in computing a physical quantity and showing that it is affected by the presence of the singularity. In the rest of this letter we shall do just that. We shall also show that, as a result of the singular nature of the propagator, the ground state changes into flat Minkowski space.

An immediate consequence of our result is that a consistent quantization around a de Sitter background requires an infrared regularization. The simplest 
choice is a term linear in the quantum field $h$ and so we add to the effective Lagrangian a term $\hat{r} h_{\mu}^{\mu}$, where $\hat{r}$ is a parameter with dimensions [mass] $]^{4}$ This regularization breaks local coordinate as well as conformal invariance and gives a mass to the graviton. However, the compensating field $\Phi(x)$ is not an independent degree of freedom. Local conformal invariance implies

$$
g_{\mu \nu} \frac{\delta S}{\delta g_{\mu \nu}}=\frac{1}{2} \Phi \frac{\delta S}{\delta \Phi}
$$

In the presence of the infrared regulator $\hat{r} h_{\mu}^{\mu}$, the equations of motions are, because of (13), inconsistent. In order to restore consistency we must also add the term $\hat{c} \Phi(x)$ with $\hat{c}$ equal to $8 \hat{r} / V$. At the end the limit $\hat{r} \rightarrow 0$ must be taken. ${ }^{10}$

Let us now expose our program: The theory, in its minimal form, contains a mass scale $\kappa^{-1}$ or, more conveniently, $V$ and two dimensionless parameters $\lambda$ and $r=\hat{r} / V^{4}$. Every physical quantity can be computed as a function of them. We shall concentrate on the curvature $R$. It is obtained by setting equal to zero the coefficient of the linear term in $h_{\mu}^{\mu}$ in the effective action. This coefficient is given by the sum of the graviton tadpole diagrams. In the tree approximation we find:

$$
R_{(0)}=24(\lambda+2 r) V^{2} \text {. }
$$

At the limit $r \rightarrow 0$ we obtain $\bar{R}$ given by Eq. (7). At higher orders we must introduce a suitable ultraviolet cut-off and, in practice, we shall use dimensional regularization. The result will be a function $R\left(V^{2}, \lambda, r, \epsilon\right)$ where $\epsilon=4-d$. The physical value is obtained by first taking the limit $r \rightarrow 0$ and then letting $\epsilon \rightarrow 0$.

This minimal theory can be easily extended to include matter couplings, if any, as well as higher derivative terms in the metric tensor. In fact, such 
terms may be desirable if we want to achieve (i) renormalizability, (ii) a bounded Euclidean action or (iii) simply to introduce more dimensionless parameters in order to obtain a consistently truncated expansion. We shall come back to this point presently.

Of course, we are not able to sum exactly the series of tadpole diagrams and obtain the exact value of $R$. Therefore, we must use some kind of approximation. One possibility is to remark that each term in the series becomes singular when $\hat{r} \rightarrow 0$, so we can try to isolate, order by order, the most singular terms. This may be possible because, in this approximation, only graviton lines contribute and both propagators and vertices become quite simple. However, in this letter we shall present, as an illustration, the results of a first order calculation with only one-loop diagrams taken into account. In order to make this truncation consistent we need one more coupling constant. The idea is similar to the one used in dimensional transmutation, ${ }^{13}$ i.e. we arrange things so that, in some small expansion parameter, one-loop diagrams become of the same order as tree diagrams and all multi-loop diagrams become of higher order. The simplest addition to Einstein's gravity which achieves our goal is the introduction of a term proportional to $R^{2}$. This term does not solve either the renormalizability or the boundedness problem, but, on the other hand, it does not spoil unitarity. The conformal extension of a theory with $R$ and $R^{2}$ terms can be written as:

$$
\begin{aligned}
\mathcal{L}= & \sqrt{-g}\left\{\frac{1}{2 \alpha^{2}}\left(\frac{\square \Phi}{\Phi}-\frac{R}{6}\right)^{2}-\frac{1}{2} g^{\mu \nu}\left(\partial_{\mu} \Phi\right)\left(\partial_{\nu} \Phi\right)-\frac{1}{12} R \Phi^{2}+\lambda \Phi^{4}\right\} \\
& +\hat{r} h_{\mu}^{\mu}+\hat{c} \Phi
\end{aligned}
$$

where $\alpha^{2}$ is a new dimensionless coupling constant and $\square \Phi$ denotes the covariant box acting on $\Phi(x)$. We prefer to work with Lagrangians containing only first 
derivatives and this can be easily achieved with the introduction of an auxiliary field $F(x) .{ }^{3}$ After some rather lengthy algebra, we rewrite (15) in the classically equivalent form:

$$
\begin{aligned}
\mathcal{L}= & \sqrt{-g}\left\{\frac{1}{2}\left(\partial_{\mu} f\right)\left(\partial_{\nu} f\right) g^{\mu \nu}-\frac{1}{2}\left(\partial_{\mu} \sigma\right)\left(\partial_{\nu} \sigma\right) g^{\mu \nu}-\frac{R}{12}\left[(v+\sigma)^{2}-f^{2}\right]\right. \\
& \left.-\frac{\alpha^{2} V^{2}}{2}\left(v_{F}+s h w \sigma+c h w f\right)^{2}\left(1+\frac{\sigma+f}{v}\right)^{2}+\lambda V^{4}\left(1+\frac{\sigma+f}{v}\right)^{4}\right\} \\
& +\hat{r} h_{\mu}^{\mu}+\hat{c} \frac{V}{v}(\sigma+f)
\end{aligned}
$$

where we have gone through the following steps of field redefinitions:

$$
\begin{aligned}
F & =-\frac{1}{\alpha^{2} \Phi^{2}}\left(\square \Phi-\frac{R}{6} \Phi\right) ; \quad \tilde{\Phi}=\Phi-F \\
F^{\prime} & =F-v_{F} ; \quad \Phi^{\prime}=\tilde{\Phi}-v_{\Phi} ; \quad V=v_{\Phi}+v_{F} \\
\left(\begin{array}{l}
\sigma \\
f
\end{array}\right) & =\left(\begin{array}{cc}
c h w & -s h w \\
-s h w & c h w
\end{array}\right)\left(\begin{array}{c}
\Phi^{\prime} \\
F^{\prime}
\end{array}\right) ; \\
s h w & =\frac{v_{F}}{v} ; \quad \operatorname{ch} w=\frac{v_{\Phi}}{v} ; \quad v=\sqrt{v_{\Phi}^{2}-v_{F}^{2}} .
\end{aligned}
$$

The Lagrangian (16) is still not very convenient for calculations because it contains non-diagonal terms bilinear in $h, \sigma$ and $f$. In order to diagonalize the quadratic forms we introduce new fields:

$$
\rho(x)=\sigma(x)+\frac{1}{3} f(x) \quad ; \quad h_{\mu \nu}^{\prime}=h_{\mu \nu}+2 \bar{g}_{\mu \nu} \frac{\sigma}{v}
$$

in terms of which there are no mixed propagators. We are finally, in a position to compute the curvature $R$, in terms of the independent parameters of our theory 
which we choose to be $\alpha^{2}, \lambda$ and $V$. In the tree approximation, the vanishing of the coefficient of the linear term in $h$ gives:

$$
-R v^{2}-12 \alpha^{2} V^{2} v_{F}^{2}+24 \lambda V^{4}+48 r V^{4}=0
$$

On the other hand, Eq. (17a) gives for $v_{F}$ :

$$
v_{F}=\frac{R}{6 V \alpha^{2}}
$$

The combination of (19) and (20) yields the value of $R_{(0)}$ given in Eq. (14). At higher orders we must compute all tadpole diagrams. The following remarks, results of a rather lengthy, although straightforward calculation, simplify this task: (i) only graviton internal lines give divergent contributions when $r$ goes to zero. (ii) For the purposes of this computation, the three-graviton coupling constant turns out to be $R v^{2}$ while each graviton propagator brings a factor $1 / v^{2}$. (iii) For perturbation theory to be meaningful we must assume that $\alpha^{2} \ll 1$. In fact, in this paper, we shall also assume that $\alpha^{2} \ll \lambda$; in this case $V^{2} / v^{2}$ is of order $\alpha^{2}$. With these remarks in mind, the higher order corrections to Eq. (19) can be written down easily. At the one-loop order we obtain:

$$
\left(A+\frac{B}{r}\right) R^{2}-R V^{2}+24 \lambda V^{4}+48 r V^{4}=0,
$$

where we have omitted terms of order $\alpha^{2}$. The term proportional to $R^{2}$ comes entirely from the one-loop calculation. We have split its contribution to two parts. The only diagram which contributes to the $B$-term, the one which diverges when $r \rightarrow 0$, is the graviton loop. All others, scalar as well as Faddeev-Popov ghost loops, contribute only to $A$. We see that, if we are allowed to truncate the loop 
expansion and solve (21) for $R$, we find, at the limit $r \rightarrow 0, R=0$. At this point the presence of the second coupling constant $\alpha^{2}$ is crucial. Indeed, one can verify that the two-loop diagrams contribute to Eq. (21) terms of order $\alpha^{2} R^{2}$ and similarly for higher loops with increasing powers of the coupling constant. It follows that, for $\alpha^{2} \ll 1$, it is consistent to keep only the terms present in Eq. (21). We conclude that, although we started with a de Sitter background space-time, quantum corrections force the curvature to vanish. de Sitter space is not a solution of the equation of motion.

Before closing we want to remark that the one-loop truncation was made only for convenience. As we mentioned earlier, one could sum the most singular terms to all orders with the same result. However, we believe that a completely nonperturbative proof should exist, similar in spirit to the ones showing the absence of spontaneous breaking of a continuous global symmetry in two dimensions, ${ }^{14}$ establishing an inequality of the form $R \leq 0$, thus excluding the case of de Sitter space.

\section{ACKNOWLEDGEMENTS}

We wish to thank S. Coleman, D. Z. Freedman, G. t'Hooft, B. Ratra and E. Tomboulis for enlightening discussions. Two of us (J.I. and T.T.) are indebted, for the hospitality shown to them, to Prof. S. D. Drell and the Theory Group at SLAC, where this work was initiated. I.A. thanks Prof. C. Bouchiat and the Laboratoire de Physique Théorique de l'Ecole Normale Superieure, where the work was completed. For continued stimulation during the intervening time we all thank Prof. G. Tiktopoulos and the Theory Group at the National Technical University of Athens. 


\section{REFERENCES}

1. See, for example, C. W. Misner, K. S. Thorne and J. A. Wheeler, in "Gravitation" (W. H. Freeman and Co., San Francisco, 1973), p. 410.

2. S. W. Hawking, Phys. Lett. 134B, 403 (1984); E. Mottola, Phys. Rev. D 31, 754 (1985) and NSF-ITP-84-159 preprint (1984).

3. I. Antoniadis, J. Iliopoulos and T. N. Tomaras, Nucl. Phys. B261, 157 (1985)

4. I. Antoniadis, J. Iliopoulos and T. N. Tomaras, preprint to appear.

5. L. H. Ford and L. Parker, Phys. Rev. D $\underline{16}, 245$ (1977); A. D. Linde, Phys. Lett. 116B, 335 (1982); S. W. Hawking and I. G. Moss, Nucl. Phys. B224, 180 (1983); S. W. Hawking, Nucl. Phys. B239, 257 (1984); B. Ratra, Phys. Rev. D 31, 1931 (1985). For a general survey of Quantum Field Theory in nontrivial background see: N. D. Birrell and P. C. W. Davies "Quantum Fields in Curved Space," Cambridge University Press, Cambridge, 1982 and references therein.

6. F. Englert, C. Truffin and R. Gastmans, Nucl. Phys. B117, 407 (1976).

7. I. Antoniadis and N. Tsamis, SLAC-PUB-3297 (1984), unpublished.

8. P. van Nieuwenhuizen, Nucl. Phys. B60, 478 (1973).

9. T. Banks, W. Fischler and L. Susskind, SLAC-PUB-3367 (1984); L. H. Ford and L. Parker, Phys. Rev. D $\underline{31}, 710$ (1985).

10. A regulator of this kind is necessary even when quantizing around flat space, where the propagator is well-defined, in order to regularize the graviton tadpole diagrams (see Refs. 11 and 12). However, in our case the role of 
the regulator is much more fundamental, because, in its absence, even the free propagator does not exist.

11. B. de Wit and R. Gastmans, Nucl. Phys. B128, 294 (1977).

12. I. Antoniadis, J. Iliopoulos and T. N. Tomaras, SLAC-PUB-3768 (1985).

13. S. Coleman and E. Weinberg, Phys. Rev. D ㄱ, 1888 (1973).

14. S. Coleman, Comm. Math. Phys. $\underline{31}, 259$ (1973); S.-K. Ma and R. Rajaraman, Phys. Rev. D 11, 1701 (1975). 